\title{
Complexity as multidimensional perception: The effects of sensory deprivation on concept identification
}

\author{
P. BRUCE LANDON and PETER SUEDFELD \\ University of British Columbia, Vancouver, British Columbia, Canada V6T IW5
}

\begin{abstract}
Task complexity was defined in terms of the number of stimulus dimensions used in a prechoice perceptual response. Simple and complex concept identification problems were presented to 24 male subjects after $22 \mathrm{~h}$ in either a sensory deprivation condition or an ambulatory control condition. The predicted complexity-arousal interaction was found with a measure of response bias but not with a measure of learning. The results were interpreted as supporting the proposed definition of complexity.
\end{abstract}

Even after many years of research, cognitive psychologists have not agreed on how to define "complexity," although the variable is frequently used in experiments. Many researchers measure complexity in terms of the variability of stimuli (e.g., number of sides or random turns in a polygon); others have used the term in relation to the open-endedness or novelty of the task to be performed (see Bartlett, 1932). One desirable development, which has not yet occurred, is to differentiate complexity from other variables, particularly difficulty. Confusion arises from the current lack of discrimination. For example, Dunn (1968) defines complexity as being related to the sequentiality of behavior, with longer chains being higher in complexity. However, this definition is essentially identical to the current usage of the term "difficulty" in the context of immediate memory load (Matarazzo, 1972).

In this study, complexity is defined in terms of the task to be performed rather than in terms of the stimulus. Specifically, the relevant indicator is the prechoice perceptual response. At the simple end of the continuum is the perception that a stimulus has properties on only one judgmental dimension; complexity increases as the stimulus is perceived as possessing more dimensions. Thus, complexity is defined as a direct function of the number of judgmental continua used by the perceiver.

A concept identification task is used because the number of usable stimulus dimensions can be easily operationalized in such a paradigm. In a series of studies (Landon \& Suedfeld, 1972; Suedfeld, 1969; Suedfeld \& Landon, 1970) it has been established that sensory deprivation interacts with task complexity as defined in Bartlett's (1932) terms. As deprivation duration increases, simple task performance is curvilinear, with the apex

This research was supported in part by an NIMH predoctoral fellowship grant to the first author, and in part by National Research Council Grant A9589 to the second author. at $24 \mathrm{~h}$. Complex task performance is best just prior to sensory deprivation and deteriorates thereafter. The present study, therefore, used sensory deprivation to evaluate the utility of the proposed perceptual dimension definition of task complexity.

\section{METHOD}

Twenty-four male undergraduates responded to campus newspaper advertisements to participate in the study for $\$ 20$. Half were assigned randomly to the sensory deprivation and half to the control condition. The experimental chamber was a sound-attenuated room, Industrial Acoustics Model 404-A. The subject lay supine on a bed in the totally dark chamber, with liquid diet food and water available ad lib at bedside. He was informed that, while he was free to leave if he wished, while he was in the room he was to stay on the bed (except when using the chemical toilet) quietly and without excessive movement.

Control subjects remained in the Psychology Building and slept in a room adjoining the experimental chamber. Both treatment conditions lasted for $22 \mathrm{~h}$. All subjects were given the same non-stress-inducing orientation to the study. The tasks were administered at the end of the treatment period in the experimental chamber with the room lights off.

The stimuli for the concept identification task were four colored lights arranged in a tight 2 by 2 cluster. For the lowcomplexity task, only one dimension, color (redundant with position), was used. The target concept was a pattern with one red and one green light shining. Feedback was given after each choice was made. The 15 other combinations of the four lights in various on and off patterns were nontarget concepts.

The high-complexity task had the same memory load as the low-complexity task, with 16 possible stimulus configurations (four bits). However, in this task the combinations varied not only in color, but also on two levels each of brightness, duration, and flicker. The stimuli varied simultaneously on four separate judgmental continua.

Each task consisted of two blocks of 30 trials each, with the target concept occurring on 15 trials. The sequence of correct choices employed for each block of trials controlled for singleand double-alternation response sequences. After the subject had completed 60 trials on one task, he was shifted to the other task for the remaining 60 trials. The order of tasks was counterbalanced across subjects. 
Table 1

Median $\beta^{*}$ as a Function of Sensory Deprivation and Complexity

\begin{tabular}{ccc}
\hline \multicolumn{1}{c}{ Treatment } & Simple Task & Complex Task \\
\hline Sensory Deprivation & 1.19 & 1.24 \\
Control & 1.43 & 1.14 \\
\hline
\end{tabular}

${ }^{*} \beta<1.0$ indicates response bias in the direction of false positives.

\section{RESULTS}

Concept identification results were analyzed with a signal detection methodology (Green \& Swets, 1966) where the subject's responses produce a discrimination score $\left(d^{\prime}\right)$ and a response bias score $(\beta)$. These scores were derived for each block of trials in each task.

An ANOVA of the d' scores showed only one reliable effect: an increase in discrimination from Block 1 $($ mean $=1.12)$ to Block $2 \quad(2.59) \quad[\mathrm{F}(1,80)=39.0$, $\mathrm{p}<.001]$. This means that virtually all subjects learned to solve both concept identification problems. This low performance ceiling may have obscured the complexity or arousal effects.

Response bias scores $(\beta)$ were analyzed by $\chi^{2}$ because of heterogeneous variance between treatment conditions $[F(5,5)=7.57, p<.025]$. The Sensory Deprivation by Complexity interaction was significant $\left[\chi^{2}(1)=4.16\right.$, $p<.05]$. Sensory deprivation resulted in less response bias than the control treatment on the simple problem, and more bias on the complex problem (see Table 1).

\section{DISCUSSION}

These findings imply that the relationship between task complexity and performance may operate through the mechanism of response bias. Response bias is presumably occasioned by the increased response competition that accompanies the increasing arousal caused by sensory deprivation. (Suedfeld, 1969). With simple unidimensional tasks, this effect improves the decision process, since fewer remote associations are made and the correct response becomes more strongly dominant. The contrary is true for complex multidimensional tasks: Increased arousal makes the decision process less efficient, since here the correct solution is not initially a high-probability response (see Spence, 1958).

In summary, the proposed definition of task complexity as a direct function of the used dimensionality of the stimulus has proved to be useful not only in uniquely characterizing complexity but also in suggesting uncovering the mechanics of the complexity-arousal relationship.

\section{REFERENCES}

BARTLETt, F. C. Remembering: $A$ study in experimental and social psychology. Cambridge: Cambridge University Press, 1932.

Dunn, J. A. Anxiety, stress, and the performance on complex intellectual tasks: A new look at an old question. Journal of Consulting and Clinical Psychology, 1968, 32, 669-673.

Green, D. M., \& Swets, J. A. Signal detection theory and psychophysics. New York: Wiley, 1966.

Landon, P. B., \& Suedfeld, P. Complex cognitive performance and sensory deprivation: Completing the U-curve. Perceptual and Motor Skills, 1972, 34, 601-602.

MATARAzZo, J. D. Wechsler's measurement and appraisal of adult intelligence (5th ed.). Baltimore: Williams \& Wilkins, 1972.

SPENCE, K. W. Theory of emotionally based drive (D) and its relation to performance in simple learning situations. American Psychologist, 1958, 13, 131-141.

SuEDFELD, P. Theoretical formulations: II. In J. P. Zubek (Ed.), Sensory deprivation: Fifteen years of research. New York: Appleton-Century-Crofts, 1969. Pp. 433-448.

Suedfeld, P., \& Landon, P. B. Motivational arousal and task complexity: Support for a model of cognitive changes in sensory deprivation. Journal of Experimental Psychology, 1970, 83, 329-330.

(Received for publication March 31, 1977.) 